\title{
Frequency Tunable Non-Reciprocal Bandpass Filter And Diplexer Using Time-Modulated Microstrip $\lambda_{g} / 2$ Resonators
}

\author{
Xiaohu Wu, Senior Member, IEEE, Mahmoud Nafe, Student Member, IEEE, Alejandro Álvarez Melcón, Senior \\ Member, IEEE, Juan Sebastián Gómez-Díaz, Senior Member, IEEE, and Xiaoguang Liu, Senior Member, IEEE
}

\begin{abstract}
This paper presents novel designs of frequency reconfigurable distributed non-reciprocal bandpass filter and diplexer based on spatio-temporally modulated microstrip $\lambda_{g} / 2$ resonators. The modulation is achieved by loading both ends of the $\lambda_{g} / 2$ transmission line resonators with time-modulated capacitors. To provide an inherent biasing isolation between the $\mathrm{RF}$ and the modulation signals, the modulation voltage source is connected at the center of the resonator, where there is a natural voltage null. A single inductor is used to further enhance such biasing isolation. The wideband nature of this isolation scheme enables the the tuning of the devices over a wide frequency range. With more than 30-dB RF to modulation isolation, the proposed resonator structure also enables low insertion loss by eliminating RF signal leakage to the modulation ports. Two examples of a 3-pole bandpass filter and a diplexer are demonstrated with good agreement between the measurement and the simulation. The fabricated filter shows a minimal insertion loss of $3.9 \mathrm{~dB}$, a 20-dB isolation bandwidth of $42 \mathrm{MHz}$ at $1.0 \mathrm{GHz}$, and frequency tuning range of $885-1031 \mathrm{MHz}$. The measured diplexer has two non-reciprocal bandpass channels at $829 \mathrm{MHz}$ and $997 \mathrm{MHz}$, respectively. The two channels can be independently reconfigured without affecting each other.
\end{abstract}

Index Terms-Bandpass filter (BPF), diplexer, distributed, isolator, microstrip, magnetic-free, non-reciprocal, time-invariant, time-modulated, tunable.

\section{INTRODUCTION}

$\mathbf{I}$ N-BAND full-duplex (IBFD) is an emerging wireless communication paradigm that has recently drawn much interest in both academic and industrial communities. IBFD allows co-located transmitters (TXs) and receivers (RXs) to operate at the same time and the same frequency, which in principle doubles the spectral efficiency and alleviates self-collision as

Manuscript received xxx xx, 2020.

This paper is an expanded version from the IEEE MTT-S International Microwave Symposium (IMS 2019), Boston, MA, USA, June 2-7, 2019.

$\mathrm{X}$. Wu is with School of Electronic and Information Engineering, Nanjing University of Information Science and Technology, Nanjing 210044, China, and also with the Department of Electrical and Computer Engineering, University of California, Davis, 95616, USA. e-mail: xhwu@ieee.org

A. A. Melcón is with the Department of Information and Communication Technologies, Technical University of Cartagena, Murcia, 30202, Spain. email: alejandro.alvarez@upct.es

M. Nafe, J. S. Gómez-Díaz, and X. Liu are with the Department of Electrical and Computer Engineering, University of California, Davis, 95616 , USA. e-mail: manafe, jsgomez, 1xgliu @ucdavis.edu well as hidden node problems [1]-[3]. In IBFD systems, nonreciprocal devices - circulators and isolators - are often used to provide the Tx-Rx isolation [4]-[6].

Conventional circulators and isolators are usually implemented with ferrite materials [7], [8]. Such devices are bulky, expensive, and difficult to integrate with other circuit blocks. Active non-reciprocal devices have been proposed with miniaturized sizes and the possibilities to be integrated with CMOS circuit processes [9]-[11]. However, their applications are limited due to low linearity, low power handling, and poor noise performance [12]. Coupled nonlinear resonances have also been used to generate non-reciprocity at both optical [13], [14] and microwave frequencies [15]. However, their operation is power-dependent, and thus inherently leads to significant signal distortion.

Recently, linear periodically time-varying (LPTV) circuits have been proposed as a new approach to achieve magneticfree non-reciprocity [16]. Depending on their implementations, LPTV circuits can be broadly categorized into 4 groups: 1) permittivity-modulated; 2) permeability-modulated; 3) resonance-modulated; 4) conductivity-modulated.

Permittivity modulation is achieved in [17], [18] by periodically loading a balanced transmission line with varactors modulated by a single tone traveling-wave of frequency $f_{m}$ and amplitude $V_{m}$. RF signal at $f_{R F}$ excited in the same direction as the modulation wave is converted to mixing products whereas signals going in the opposite direction will pass through the transmission line. Non-reciprocity is obtained at the mixing frequency $f_{m}-f_{R F}$ and thus extra duplexers are needed to separate the TX and RX signals $\left(f_{R F}\right.$ and $f_{m}-f_{R F}$, respectively) to exhibit a circulator performance [17]. The detailed analysis in [17] shows that to have a full conversion in the forward direction, the minimum optimum transmission line length of $\lambda_{g} /(\sqrt{2} \xi)$ is needed, where $\lambda_{g}$ is the guided wavelength at $f_{R F}$ and $\xi$ is the capacitance modulation index. This results in a large circuit size. Permittivity modulation in the form of angular-momentum biasing of a resonant ring also achieves strong non-reciprocal circulator responses [19]. Permeability modulation has been investigated by using Josephson junctions as nonlinear inductors [20].

Instead of modulating the permittivity or permeability in a continuous fashion, Estep et al. modulates three lumpedelement $L C$ resonators connected in either a $\Delta$ or $Y$ topology to reduce the circuit size [21], [22]. Several extensions of this 
concept are reported in [23]-[28]. It is also shown that the linearity of such circulators can be improved by combining two circulators in a differential fashion [29] at the cost of doubling the circuit size and numbers of components.

Conductivity-modulated circulator circuit is first reported in a CMOS process in [30]. In this work, a staggered commutation network with non-reciprocal $\pi$ phase shift is combined with a $3 \lambda_{g} / 4$ transmission-line loop. Asymmetrical circuit configuration is used to enhance the TX-Antenna power handling but at the penalty of impedance mismatches at the ports. Besides, the circuit needs the switching frequency $f_{m}$ to be the same with the non-reciprocal center frequency $f_{R F}$. resulting in challenges in the power consumption and leakage suppression between $f_{m}$ and $f_{R F}$. In [31], a non-reciprocal isolator consisting of a delay line sandwiched between two time-modulated resistors is presented. A gyrator with a nonreciprocal phase difference of $\pi$ is also realized and applied to implement a fully integrated passive circulator in a 45 $\mathrm{nm}$ silicon-on-insulator (SOI) CMOS [31]-[33], where the modulation (switch) frequency is lowered to one-third of the non-reciprocal frequency. The same idea is applied to implement an RF non-reciprocal gyrator and circulator based on switched acoustic delay lines in [34].

In [35], we demonstrate a novel non-reciprocal bandpasss filter using spatio-temporal modulation of a lumped-element coupled-resonator filter. A full-spectra domain method is developed to analyze and synthesize the time-varying network properties. We show that the same-frequency non-reciprocity is a result of the non-reciprocal frequency conversion to and from the inter-modulation (IM) frequencies by the timevarying resonators. The analysis of such filters is generalized to a time-varying coupling matrix approach in [36]. To verify the analysis, non-reciprocal filters based on time-modulated microstrip resonators are designed. Although good correlation between the theoretical and measured results is observed, the complicated biasing design as well as the cross coupling effect lead to sub-optimal filter responses (more details will be given in Section III-D.

As an alternative solution, a 2-pole distributed nonreciprocal filter based on time-modulated $\lambda_{g} / 2$ resonators is reported in [37]. The modulation sources are directly loaded to the $\mathrm{RF}$ resonators, leading to a weak $f_{m}-f_{R F}$ isolation of around $10 \mathrm{~dB}$. As a result, spurious even-mode resonances deteriorate the filtering performances.

In this paper, we extend the work of [37] in the following aspects:

1) Detailed analysis of the biasing/modulation scheme has been given and validated in a new 3-pole non-reciprocal filter design. We demonstrate that with the help of a single inductor, an inherent and wideband $f_{m}-f_{R F}$ isolation of better than $30 \mathrm{~dB}$ can be achieved by taking advantage of the voltage null point near the center of $\lambda_{g} / 2$ resonators. The high $f_{m}-f_{R F}$ isolation not only eliminates the spurious resonances but also results in less RF leakage and lower forward insertion loss.

2) A non-reciprocal diplexer with integrated functions of a conventional bandpass diplexer and an isolator is presented. To the best of the authors' knowledge, this

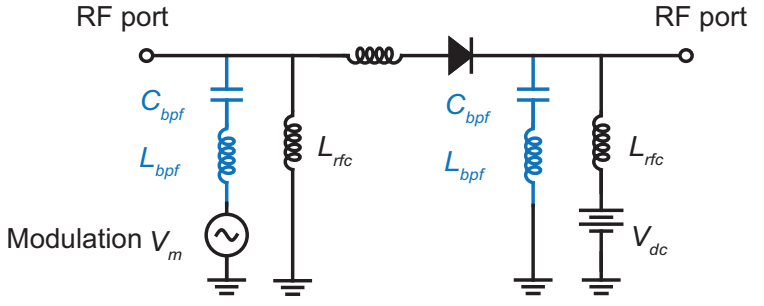

(a)

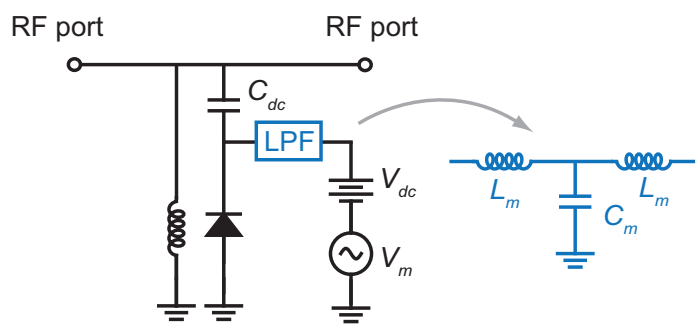

(b)

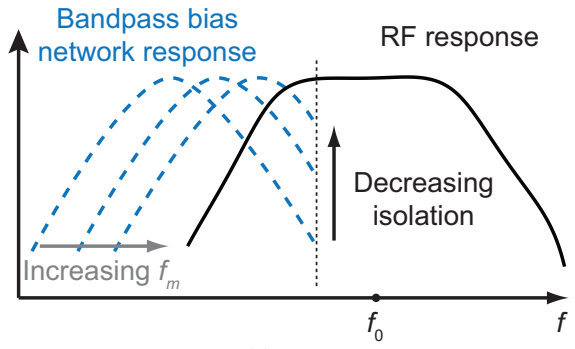

(c)

Fig. 1. Reported varactor-based time-varying resonators. (a) Time-varying serial $L C$ resonator with individual BPF and dc bias circuits. (b) Time-varying parallel $L C$ resonator with LPF bias circuit and a single dc block capacitor $C_{d c}$. (c) Bias limitation as larger $f_{m}$ is increased. $f_{0}$ is the RF passband center frequency.

is the first time that a non-reciprocal diplexer has been presented.

3) Owing to the wideband nature of the biasing/modulation scheme, we demonstrate frequency tuning capabilities in both the non-reciprocal filter and the non-reciprocal diplexer designs.

The rest of this paper is organized as follows. In Section[I] distributed time-varying resonator is described. First, a review of the reported time-varying resonators is provided to elaborate on their limitations. Subsequently, distributed time-varying resonator and its static as well as time-varying properties are presented. The bias setup for the proposed time-varying resonator is also discussed in detail. Section [II] demonstrates the experimental results of a non-reciprocal filter and diplexer. The frequency reconfigurability of both designs is also examined in section III-C. Section III-D compares its performance with the state-of-art. Finally, a conclusion is given in SectionIV

\section{TIME-VARYing RESONATORS}

\section{A. Review of Reported Time-Varying Resonators}

Existing works on spatio-temporal modulation (STM) based non-reciprocal components are predominantly based on modulating lumped-element resonators [21], [22], [35]. Both series 


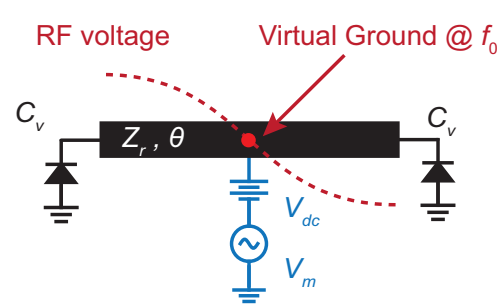

(a)

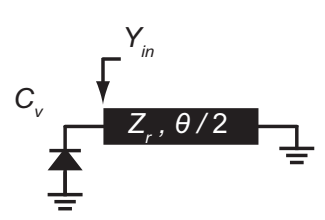

(b)

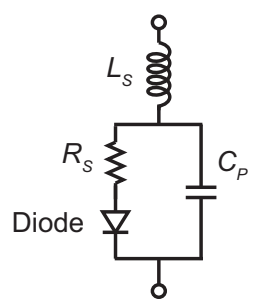

(c)

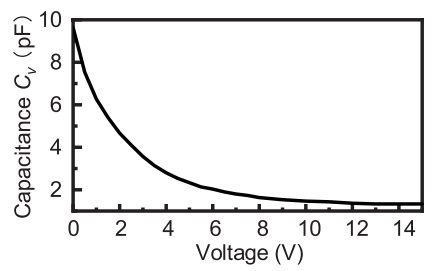

(d)
Fig. 2. (a) Resonant structure of the proposed time-varying microstrip $\lambda_{g} / 2$ resonator. $C_{v}$ is the equivalent capacitance of the reversely biased varactor. $f_{0}$ is the resonance frequency and also the filter passband center frequency. (b) Odd-mode equivalent circuit. (c) The spice model of the loaded varactors. (d) Nonlinear $C-V$ curve of the varactors.

resonators [Fig.1](a)] and shunt resonators [Fig.11(b)] have been used. Solid-state diode varactors together with a static inductor are used to create a time-varying resonance.

As illustrated in Fig. 1. a key design challenge in these timevarying resonators lies in separating the RF and modulation signals. This is usually achieved by duplexing circuits. In Fig. 11(a), for example, $C_{b p f}$ and $L_{b p f}$ constitute a bandpass filter centered at the modulation frequency $f_{m}$ to prevent the RF signal from leaking into the modulation voltage source. Because the bandpass filter also blocks the dc bias voltage, an additional dc return pass is provided by a large inductor $L_{r f c}$ as an RF choke. Similarly, in Fig.11(b), the modulation voltage source is isolated by a low-pass filter and a static dc-blocking capacitor $C_{d c}$. Obviously, in a practical implementation, the addition of the bias and duplexing circuits will add to the insertion loss of the overall circuit.

In addition, it is important to realize that $f_{m}$ is approximately equal to the inherent static RF bandwidth $B W$ of the non-reciprocal filter [35]. A larger static $B W$ together with higher $f_{m}$ can provide wider non-reciprocal bandwidth. As a result, the cut-off frequency of the duplexing circuit must also be increased to accommodate the larger $f_{m}$, which in turn will reduce the rejection level at the RF frequency. Taking the bandpass biasing case for an example, as shown in Fig.11(c), the bandpass biasing attenuation at the nonreciprocal passband frequency $f_{0}$ decreases with the increase of $f_{m}$, leading to more $R F$ leakage to the modulation sources. The same situation can be found in the lowpass bias case. This can be solved by increasing the order of the biasing bandpass or lowpass filters to have higher roll-off, although at the cost of increased circuit complexity and higher insertion loss of the overall circuit.

Lastly, lumped inductors and capacitors are known to have relatively low quality factor $(Q)$ and high parasitics that limit their use to relatively low frequencies.

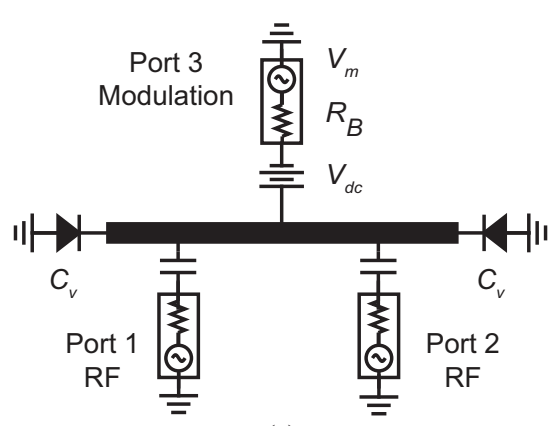

(a)

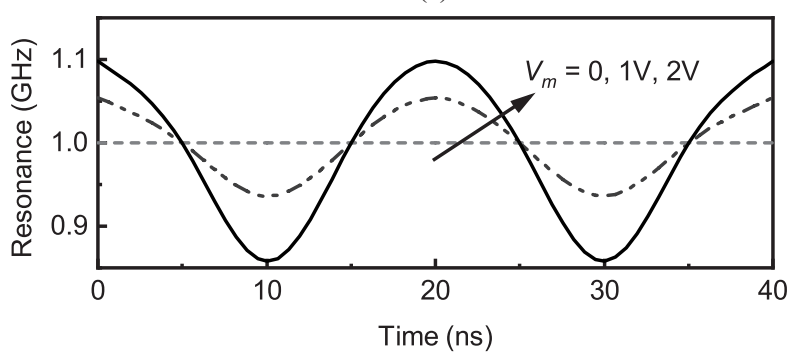

(b)

Fig. 3. (a) Proposed time-modulated resonator with weak external coupling to extract the time-variant resonant frequency. (b) Extracted time-varying resonant frequency with respect to the modulation amplitude $V_{m}$. Here, $V_{d c}=6.45 \mathrm{~V}, V_{m}=1.0 \mathrm{~V}$, and $R_{B}=50 \Omega$.

\section{B. Proposed Distributed Time-Varying Resonator}

To overcome the difficulties of lumped-element resonators in terms of biasing, loss, and parasitics, we propose a distributed time-varying resonator as shown in Fig.2. To modulate the resonant frequency, the half-wavelength $\left(\lambda_{g} / 2\right)$ resonator is symmetrically loaded by varactors at both two ends. The modulating wave is applied at the center of the resonator which is a virtual ground at $f_{R F}$ but not at $f_{m}$. As a result, the proposed time-varying distributed resonator can realize inherent isolation between the RF signal and the modulation signal, besides having higher $\mathrm{Q}$ and lower parasitics than lumped-element resonators.

1) Resonant frequency: Due to the symmetry of the $\lambda_{g} / 2$ resonator, its resonant frequency can be analyzed by its oddmode circuit shown in Fig.2(b). The input admittance to the odd-mode circuit is

$$
Y_{i n}=j\left(\omega C_{v}-Y_{r} \cot \frac{\theta}{2}\right) .
$$

The resonant frequency $f$ can be solved by setting $I_{m}\left\{Y_{i n}\right\}=0$,

$$
f=\frac{Y_{r}}{2 \pi C_{v}} \cot \frac{\theta}{2} .
$$

In this work, an 80- $\Omega$ transmission line with $\theta=80^{\circ}$ and an equivalent static capacitance of $2.35 \mathrm{pF}$ are chosen for a nominal (static) resonant frequency of $1.0 \mathrm{GHz}$. Skyworks SMV1234 varactors are used to modulate the resonator. The nominal capacitance of $2.35 \mathrm{pF}$ is achieved at a reversely biased $d c$ voltage of $5.5 \mathrm{~V}$ [38]. Fig.2(c) shows the equivalent circuit model of the varactor and Fig.2(2) shows its $C-V$ characteristics.

The resonant frequency of the resonator can be modulated by modulating the varactors with a sinusoidal voltage $V_{m}$. 


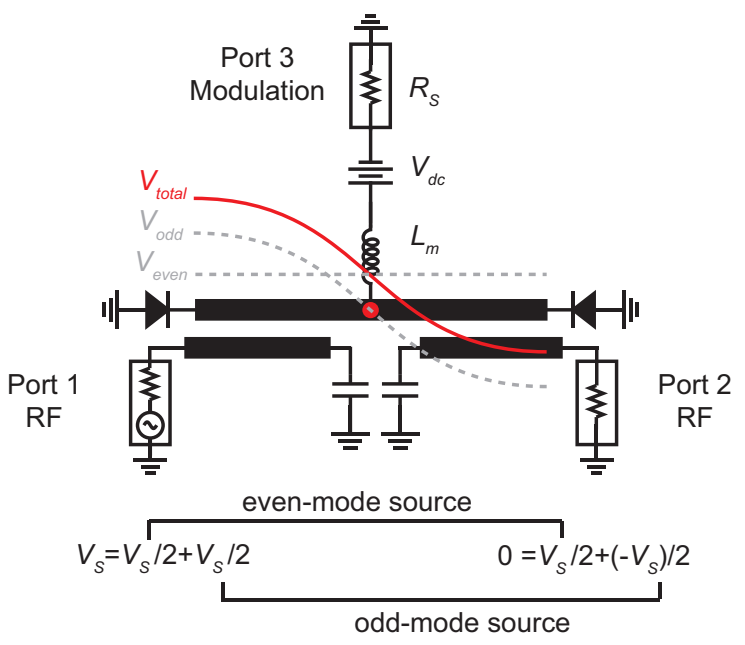

(a)

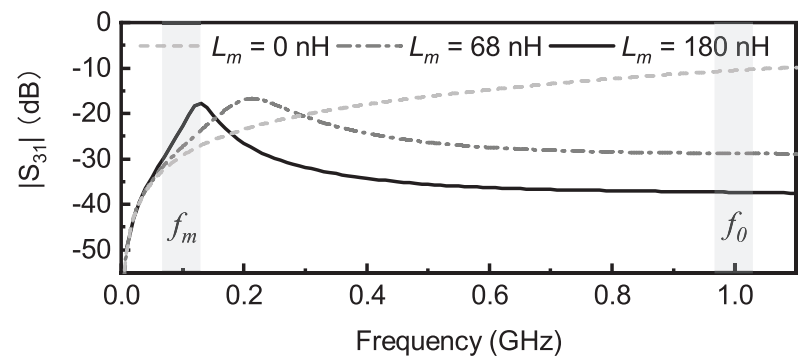

(b)

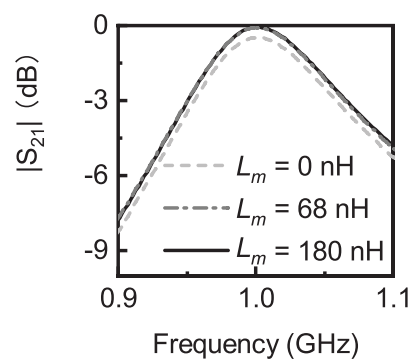

(c)

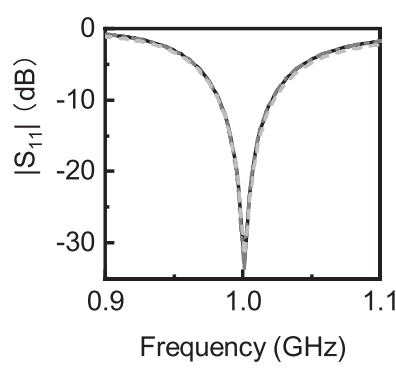

(d)
Fig. 4. (a) Circuit simulation setup to study the $f_{m}-f_{R F}$ isolation of the timevarying resonator. $V_{\text {even }}, V_{\text {odd }}$, and $V_{\text {total }}$ are the even mode, odd mode, and total waveform, respectively. (b) Simulated $f_{m}-f_{R F}$ isolation (|S $S_{31} \mid$ ). (c) Simulated RF transmission $\left|S_{21}\right|$. (d) Simulated RF reflection $\left|S_{11}\right|$. Legend of (d) is removed for clarity, lines correspond to the same investigated inductor values as (b) and (c).

Fig.3 (a) shows the simulation setup to study the time-varying resonant properties of the proposed resonator. The resonator is weakly coupled to the source and the load by two $0.03-\mathrm{pF}$ capacitors. Harmonic balance in Keysight Advanced Design System (ADS) is used to extract the time-varying resonant frequency [Fig.3(b)]. Here, a 50-MHz sinusoidal wave with phase $\phi=0^{\circ}$ and various amplitude $V_{m}$ is used as the modulation waveform. The resonance is time-invariant when $V_{m}=0 \mathrm{~V}$, which is the case without time-modulation. Higher $V_{m}$ results in a larger modulation on the resonance. Note that the resonant frequency is nonlinear with respect to the modulation waveform due to the nonlinearity of (2) and the varactor's nonlinear $C-V$ characteristics.

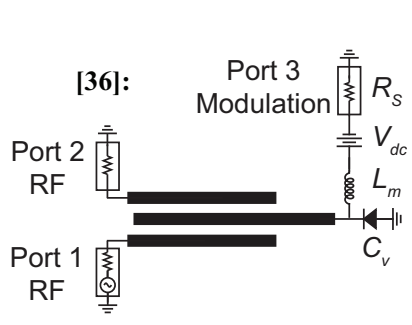

(a)

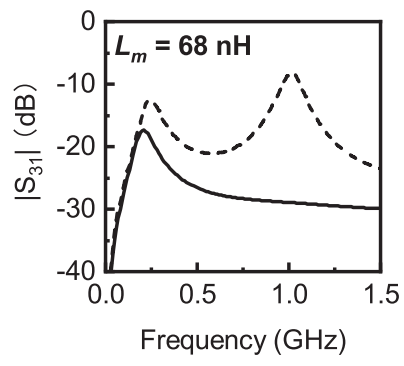

(c)

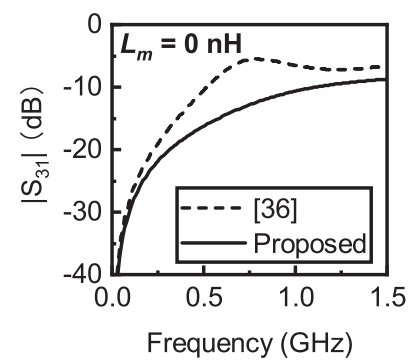

(b)

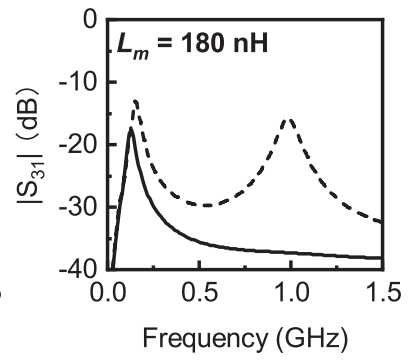

(d)
Fig. 5. Comparison studies between the proposed work and work in [36]. (a) Time-modulated resonator in [36]. Compared biasing isolation when (b) $L_{m}=0 \mathrm{nH}$, (c) $L_{m}=68 \mathrm{nH}$, and (d) $L_{m}=180 \mathrm{nH}$. The Legends of (c) and (d) are the same as (a) and are removed for clarity.

2) Bias isolation: With the modulation circuitry connected to the center of the $\lambda_{g} / 2$ resonator, an inherent isolation between the bias signal and the RF signal is provided. If the resonator is under an odd mode excitation, the RF signal is ideally isolated from the bias signal due to the symmetry of the structure [Fig.2(a)]. In an actual filter, however, the resonators are usually not excited with an ideal differential waveform. The setup of Fig. 4(a) is used to understand the $f_{m^{-}}$ $f_{R F}$ isolation through S-parameter simulations. In S-parameter simulation, all ports are matched while one of the ports (e.g., Port 1) is excited with a source. As such, the excitation is a combination of odd mode and even mode sources. Due to the existence of the even mode effect, the voltage on the $\lambda_{g} / 2$ resonator is not ideally symmetric, leading to a nonperfect isolation. In fact, Fig. 4 (b) shows that only $10-\mathrm{dB} f_{m^{-}}$ $f_{R F}$ isolation can be achieved at $f_{0}$. As seen in [37], such a weak biasing isolation results in a strong loading effect of the modulation circuit on the even-mode resonances and introduces undesired spurious responses.

To provide an enhanced isolation, additional low-pass filter can be inserted at the modulation port. A simple implementation takes the form of a series inductor $L_{m}$. Fig. 4(b) shows the $f_{m}-f_{R F}$ isolation for various $L_{m}$ values. An $L_{m}$ value of $68 \mathrm{nH}$ can improve the isolation at $f_{R F}$ by almost $20 \mathrm{~dB}$. Isolation at $f_{m}$ remains almost the same at $35 \mathrm{~dB}$. Note that $\left|S_{31}\right|$ is equal to $\left|S_{32}\right|$ due to the symmetry and that the isolation between both the RF ports and the modulation port is very good. The enhanced $f_{m}-f_{R F}$ isolation also leads to lower $R F$ transmission insertion loss between the two $R F$ ports. As seen in Fig.4(c) and (d), the model in Fig.4(a) has a passband centered at $1.0 \mathrm{GHz}$. Due to the $f_{R F}$ leakage to the modulation branch, $0.66-\mathrm{dB}$ insertion loss can be observed 
from the filter transmission coefficient $\left|S_{21}\right|$ when $L_{m}=0$. With an inductor $L_{m}$ of $68 \mathrm{nH}$, a $0.38-\mathrm{dB}$ improvement of insertion loss can be attained.

Although a small $f_{m}$ value is used in this work, the depicted isolation behaviour of Fig.4(b) indicates that larger $f_{m}$ values are also as effective. This property may benefit other applications, such as high-performance circulators. For example, STM circulators using larger higher $f_{m}$ have lower transmission insertion loss, smaller intermodulation products, better port matching, and larger separation between the desired RF signal and the intermodulation products [22].

Note that distributed time-modulated resonator was also presented in [36]. For illustration purpose, it is re-produced in Fig.5 (a) with input/output couplings. A direct comparison between the biasing schemes of this work and that of [36] is presented in Fig.5(b), (c), and (d). To make a fair comparison, the time-varying resonators are designed at the same resonant frequency $1.0 \mathrm{GHz}$. It can be clearly seen that the $f_{m}-f_{R F}$ isolation of the biasing scheme in this work is significantly better than that of [36].

Similar to previous works in STM-based non-reciprocal circuits ( [21], [22], [35]), the $f_{m}-f_{R F}$ isolation in [36] solely relies on the indcutor $L_{m}$. In contrast, this work takes advantage of the virtual ground in $\lambda_{g} / 2$ resonator to present a biasing scheme that is much more effective than [36] in terms of $f_{m}-f_{R F}$ isolation, isolation bandwidth, and simplicity.

\section{ApPliCATIONS AND RESUlts}

Non-reciprocal bandpass filter and diplexer using the proposed time-modulated $\lambda_{g} / 2$ resonators are presented in this section. The filter and diplexer are designed on Rogers 4003C substrates with a thickness of $0.813 \mathrm{~mm}$, a relative permittivity of 3.55, and a loss tangent of 0.0027. As illustrated in [35], the design of non-reciprocal filter can start with designing a conventional reciprocal filter of static specifications. The final non-reciprocal filtering performances can be achieved by applying proper time-modulation with a modulation phase difference $(\Delta \varphi)$ between the adjacent resonators. The closeto-optimum modulation is found to have $\Delta \varphi=45^{\circ}$ and $f_{m} \simeq B W[35]$.

\section{A. Non-Reciprocal Filter And Results}

The design of a 3-pole non-reciprocal filter of the in-line coupled diagram in Fig.6(a) statrs with a conventional static and reciprocal 3-pole filter [35]. The corresponding static filter is specified to be centered at $1.0 \mathrm{GHz}$ with a Chebyshev response having a passband ripple of $0.04 \mathrm{~dB}$ and a ripple bandwidth of $60 \mathrm{MHz}$. The corresponding lowpass prototype filter has element values of $g_{0}=1.0, g_{1}=0.8516, g_{2}=$ $1.1032, g_{3}=0.8516$, and $g_{4}=1.0$. The design parameters, namely the coupling coefficient $M_{i j}$ and the external quality factor $Q_{e}$, can be obtained as follows [39]

$$
\begin{aligned}
M_{12} & =\frac{F B W}{\sqrt{g_{1} g_{2}}}=0.062, M_{23}=\frac{F B W}{\sqrt{g_{2} g_{3}}}=0.062, \\
Q_{e} & =\frac{g_{0} g_{1}}{F B W}=\frac{g_{3} g_{4}}{F B W}=14.2 .
\end{aligned}
$$

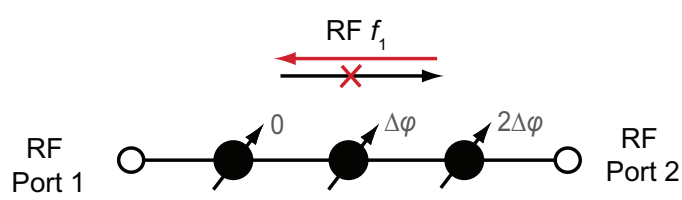

(a)

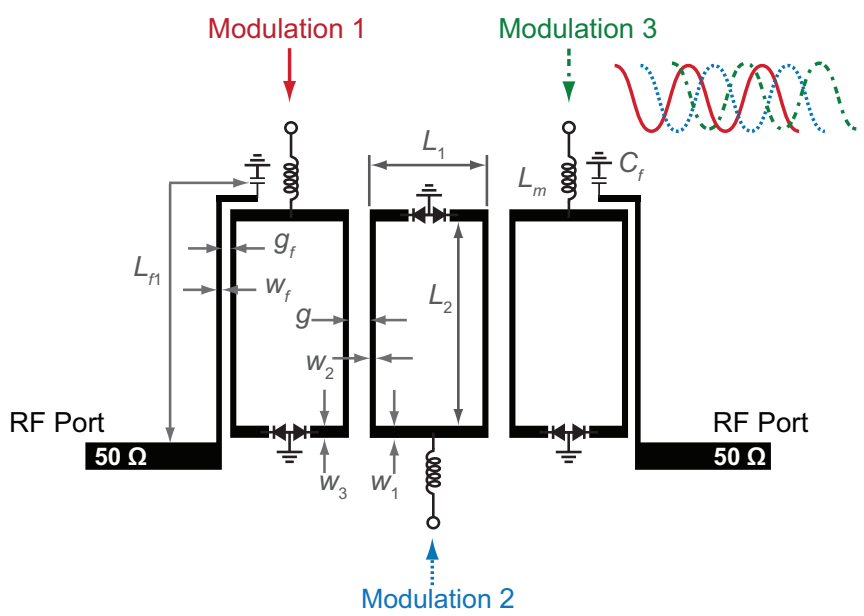

(b)

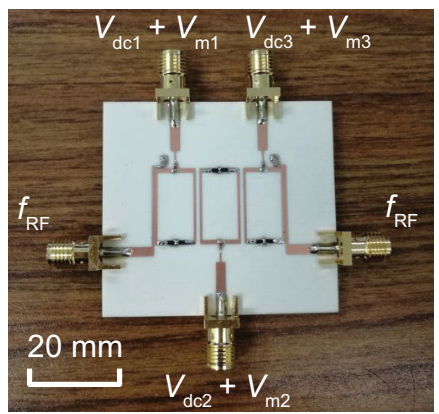

(c)

Fig. 6. Proposed 3-pole microstrip filter. (a) Coupling diagram. White circles: source (S) and load (L); Solid lines: coupling; Black circles with arrows: timemodulated resonators. (b) Microstrip layout $\left(C_{f}=2.2 \mathrm{pF}\right.$ and $\left.L_{m}=68 \mathrm{nH}\right)$. (c) Photo of the fabricated 3-pole non-reciprocal BPF.

According to the analysis in Section II a characteristic impedance of $80 \Omega$ is chosen for the microstrip resonator. An electrical length of $80^{\circ}$ and loaded equivalent capacitance of $2.35 \mathrm{pF}$ are chosen for a center frequency of $1.0 \mathrm{GHz}$.

The layout of the 3-pole filter is shown in Fig.6(b). The three time-modulated microstrip resonators of ring configuration are in-line coupled to each other. The resonator strip width along the coupling side is slightly reduced to provide larger coupling to meet the bandwidth requirement. The dimensions of the resonators are $L_{1}=7.80 \mathrm{~mm}, L_{2}=13.42 \mathrm{~mm}$, $w_{1}=0.80 \mathrm{~mm}, w_{2}=0.40 \mathrm{~mm}$, and $w_{3}=0.80 \mathrm{~mm}$. The varactors are reversely biased at $6.4 \mathrm{~V}$ to provide an equivalent static capacitance of $2.0 \mathrm{pF}$.

The dimensions of the coupling structures, $g$ for $M_{12}\left(M_{23}\right)$ and $L_{f 1}, g_{f}$, and $w_{f}$ for $Q_{e}$, can be extracted by conventional filter design method [39]. $g$ is chosen to be $0.77 \mathrm{~mm}$ to realize an $M_{i j}$ of $0.062 . w_{f}=0.4 \mathrm{~mm}, L_{f 1}=18.3 \mathrm{~mm}$, and $g_{f}=$ $0.17 \mathrm{~mm}$ are chosen to realize a $Q_{e}$ of 14.2 .

A photo of the fabricated 3-pole filter is shown in Fig.6(c). 


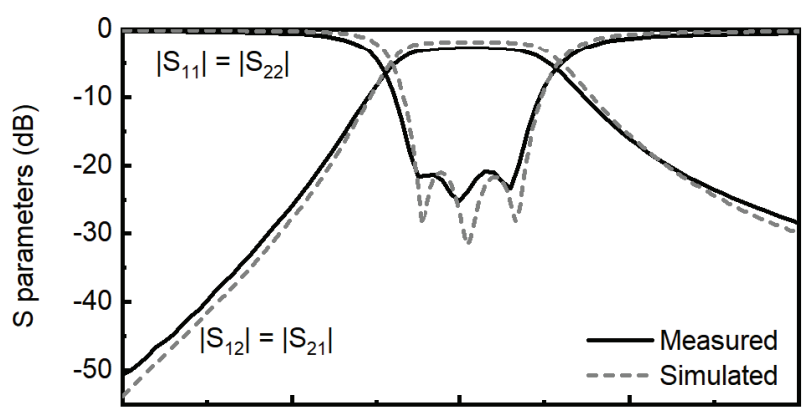

(a)

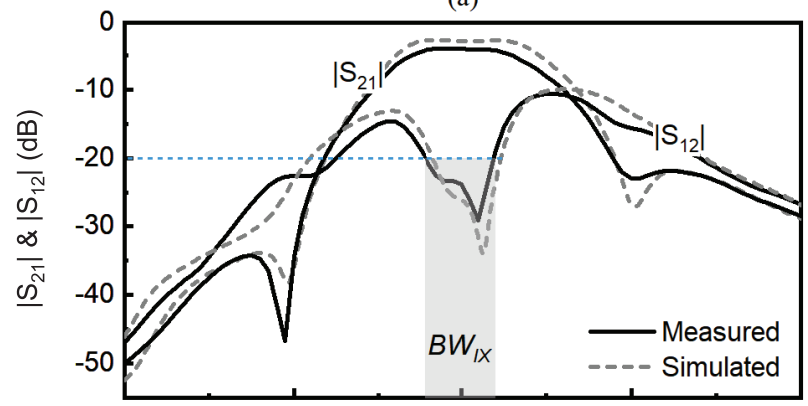

(b)

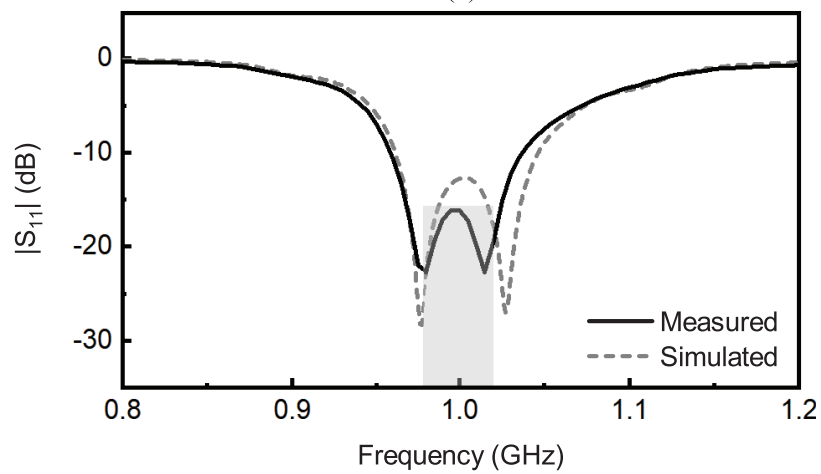

(c)

Fig. 7. Results of the 3-pole non-reciprocal microstrip filter. (a) Static responses. (b) Non-reciprocal transmission $\left|S_{21}\right|$ and $\left|S_{12}\right|$ under proper modulation. $B W_{I X}$ refers to the $20 \mathrm{~dB} I X$ bandwidth. (c) $\left|S_{11}\right|$ of the non-reciprocal filter.

The filter occupies an area of $25 \mathrm{~mm} \times 18 \mathrm{~mm}\left(0.14 \lambda_{g} \times\right.$ $0.1 \lambda_{g}$, where $\lambda_{g}$ is the guided wavelength at the center frequency).

The simulated and measured results of the static 3-pole filter are shown in Fig.77(a). Without modulation, the static filter exhibits a conventional reciprocal 3-pole Chebyshev passband at $1.0 \mathrm{GHz}$, which is close to its static specifications. The measured minimum insertion loss is $2.6 \mathrm{~dB}$ which is mainly due to the loss of the varactors. The measured transmisson $3-\mathrm{dB}$ bandwidth is $959-1055 \mathrm{MHz}$ (fractional bandwidth of $9.6 \%$ ). Moreover, the measured return loss is better than $20 \mathrm{~dB}$ across the passband.

Next, to achieve a non-reciprocal bandpass filter performance, time-modulation with incremental phase $\Delta \varphi=45^{\circ}$ is applied. The resonant frequencies of the resonators are modulated by applying sinusoidal waves to the loading varactors. The modulation phase difference between adjacent resonators is $45^{\circ} . f_{m}$ and $V_{m}$ are optimized to be $65 \mathrm{MHz}$ and $1.5 \mathrm{~V}$, respectively. The resulting non-reciprocal responses are
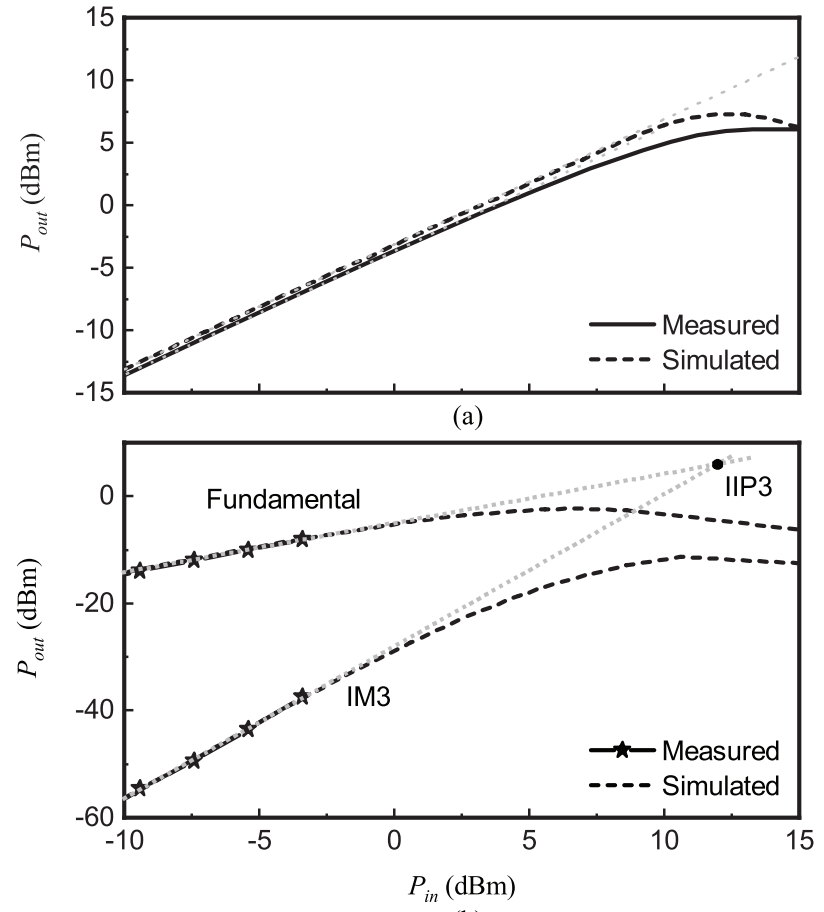

(b)

Fig. 8. Power handling and nonlinearity measurement of the fabricated 3pole non-reciprocal filter. (a) Simulated and measured P1dB with a single-tone signal at $1.0 \mathrm{GHz}$. (b) Simulated and measured IIP3 with a two-tone signal centered at $1.0 \mathrm{GHz}$ and separated by $20 \mathrm{MHz}$.

shown in Fig.77(b) and Fig.7/(c), where two isolation poles and two reflection poles can be observed in both simulation and measurement. Close agreement between the simulated and measured responses can be observed. The measured 3$\mathrm{dB}$ transmission bandwidth of the modulated filter is 958 $1044 \mathrm{MHz}$, i.e., $B W_{3 d B}=86 \mathrm{MHz}$. The measured forward insertion loss $\left(\left|S_{21}\right|\right)$ is $3.9 \mathrm{~dB}$, which is $1.1 \mathrm{~dB}$ higher than the static case. This is mainly due to the power conversion to the other intermodulation (IM) frequencies. The measured backward isolation (IX) [35] is $23.2 \mathrm{~dB}$ at the center frequency $1.0 \mathrm{GHz}$. The measured $20-\mathrm{dB}$ IX bandwidth $\left(B W_{I X}\right)$ is $978-$ $1020 \mathrm{MHz}$, i.e., $B W_{I X}=42 \mathrm{MHz}$. Note that $B W_{I X}$ is smaller than transmission bandwidth $B W_{3 d B}$. This is generally true in other STM-based circulator/isolator works [21][29], [35], [36]. The non-reciprocal bandwidth is bounded by the inherent static bandwidth and $2 f_{m}$ [28]. Beyond the 20$\mathrm{dB}$ isolation bandwidth the filter show weak non-reciprocity. For example, $\left|S_{12}\right|$ at $1050 \mathrm{MHz}$ is $-10 \mathrm{~dB}$ and it is close to $\left|S_{21}\right|$. The measured return loss across the $20-\mathrm{dB}$ isolation bandwidth is better than $16.1 \mathrm{~dB}$.

The filter has a static Chebyshev response [Fig.77(a)] as specified. Different from the work in [36], there is no deterioration at the upper band-edge transmission because a true in-line coupled topology without cross coupling is achieved in this design. Interestingly, its corresponding modulating state becomes a quasi-elliptic response with two transmission zeros at $895 \mathrm{MHz}$ and $1100 \mathrm{MHz}$, respectively. These two transmission zeros are the results of "cross coupling" in the time-modulated network. Here, unlike in conventional filter 
designs that cross coupling is introduced by two or more intentionally designed physical paths, the cross coupling in a time-modulated network is created because of the RF signal cancellation between these multiple equivalent conversion paths (RF-IM-RF) and the main RF-RF transmission path.

The power handling of the non-reciprocal filter is mainly limited by the nonlinearity of the varactors. Fig. 8 shows the simulated and measured input-referred 1-dB compression point $(\mathrm{P} 1 \mathrm{~dB})$ and the input-referred third-order intercept point (IIP3). The IIP3 measurement is done with two signals at $990 \mathrm{MHz}$ and $1010 \mathrm{MHz}$. The measured $\mathrm{P} 1 \mathrm{~dB}$ is $9.2 \mathrm{dBm}$ and the measured IIP3 is $11.8 \mathrm{dBm}$. The linearity and power handling of the non-reciprocal filter may be improved by using stacked diodes configurations [40], [41].

\section{B. Non-Reciprocal Diplexer And Results}

In this subsection, the first demonstration of a non-reciprocal diplexer is presented by using the proposed time-modulated $\lambda_{g} / 2$ resonators. The diplexer integrates functions of an isolator and a conventional bandpass diplexer. Fig.9(a) illustrates the coupling diagram of the proposed diplexer. It has two filtering channels operating at RF frequency $f_{1}$ and $f_{2}$, respectively. Both channels are composed of in-line coupled resonators, whose resonant frequencies are modulated by sinusoidal waves of incremental modulation phase $\Delta \varphi$. By setting $\Delta \varphi=45^{\circ}$, the RF signal power at $f_{1}$ and $f_{2}$ can be transmitted from the input port 1 to the output ports 2 and 3, respectively. Note that due to the odd-symmetry of spatio-temporal modulation in filtering networks [35], the transmission direction in Fig.9](a) can be readily interchanged by setting $\Delta \varphi=135^{\circ}$.

Similar to the design of non-reciprocal filters, the design of the non-reciprocal diplexer starts from its corresponding static state. Non-reciprocal performance can then be obtained by applying the proper modulation. In this design example, the two channels are specified at $835 \mathrm{MHz}$ and $1.0 \mathrm{GHz}$, respectively. The static states of both channels exhibit a reciprocal Chebyshev response having a passband ripple of $0.04 \mathrm{~dB}$ and an equal ripple bandwidth of $33 \mathrm{MHz}$. Following the resonator analysis in Section III and the coupling analysis in Section III-A. Fig.9 (b) shows the microstrip layout of the implemented diplexer using the varactor-loaded $\lambda_{g} / 2$ resonators. The varactors loaded to the left hand side of Port 1 are reversely biased at $5.9 \mathrm{~V}$, while those loaded to the right hand side are reversely biased at $6.3 \mathrm{~V}$. Fig.9 (c) shows a photo of the fabricated non-reciprocal diplexer. It occupies an area of $64 \mathrm{~mm} \times 23 \mathrm{~mm}\left(0.3 \lambda_{g 1} \times 0.1 \lambda_{g 1}\right.$, where $\lambda_{g 1}$ is the guided wavelength at the center frequency $835 \mathrm{MHz}$ of channel 1).

The static responses of the diplexer are shown in Fig.10(a) in the next page. Channel 1 and channel 2 have reciprocal filtering passband at $835 \mathrm{MHz}$ and $998 \mathrm{MHz}$, respectively. The measured insertion loss is $3.8 \mathrm{~dB}$ for channel 1 and $4.5 \mathrm{~dB}$ for channel 2. The measured transmission $3-\mathrm{dB}$ bandwidth of the two channels are $56 \mathrm{MHz}$ and $53 \mathrm{MHz}$, respectively. Despite using the same resonator structure, the insertion loss of channel 2 of the diplexer [Fig. 10(a)] is about $1.9 \mathrm{~dB}$ higher than that of the 3-pole filter [Fig.77(a)] due to the smaller bandwidth of the diplexer channels. The measured return loss

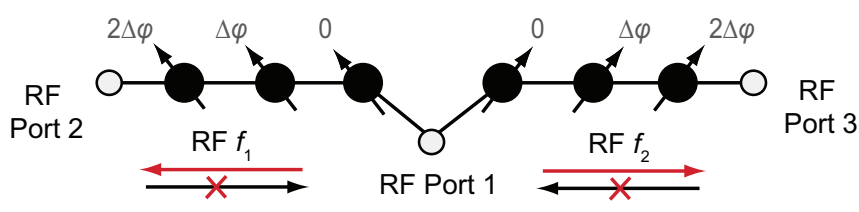

(a)

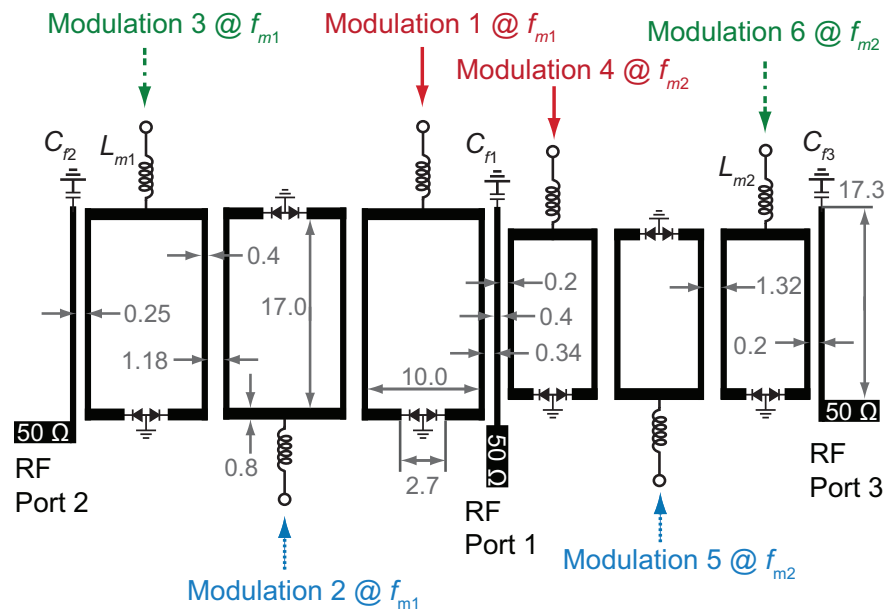

(b)

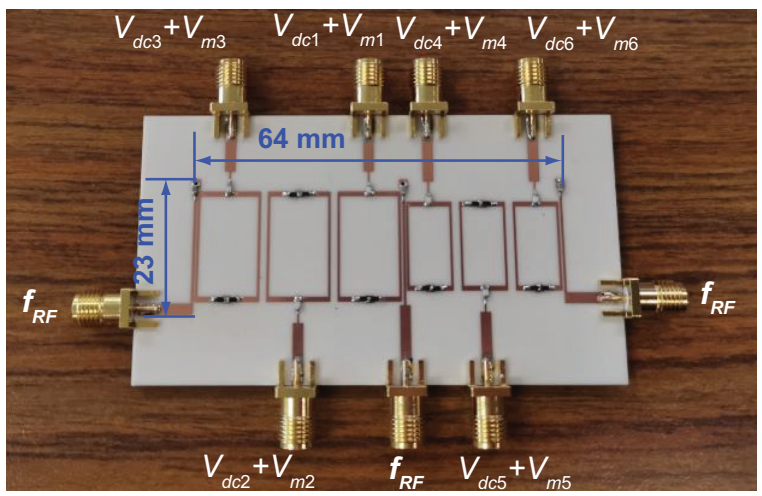

(c)

Fig. 9. (a) Coupling diagram of the 3-pole diplexer. (b) Layout of the 3pole microstrip non-reciprocal diplexer $\left(C_{f 1}=C_{f 2}=C_{f 3}=2.2 \mathrm{pF}\right.$ and $L_{m 1}=L_{m 2}=68 \mathrm{nH}$ ). (c) Photo of the fabricated diplexer.

across the two channels is better than $19 \mathrm{~dB}$ [Fig.10(a)]. More than 42-dB all-band isolation between the two output ports is obtained [Fig.10(a)]. The achieved high output port isolation is the result of resonance discrepancies between the two channels. As we will see in Section. III-C, such high channel isolation allows an independent design of spatiotemporal modulation parameters for the diplexer channels.

To realize non-reciprocity in the diplexer, time-modulation with $\Delta \varphi=45^{\circ}$ is applied to the three resonators of channels 1 and 2. The optimized modulation parameters for channel 1 are $f_{m}=45 \mathrm{MHz}$ and $V_{m}=0.9 \mathrm{~V}$, while those of channel 2 are $f_{m}=42.5 \mathrm{MHz}$ and $V_{m}=0.75 \mathrm{~V}$. The simulated and measured responses of the non-reciprocal diplexer are compared in Fig. 10(b)-(d). Both channels of the diplexer show obvious non-reciprocal filtering performances. Two distinctive isolation poles at the reverse attenuation profiles of 


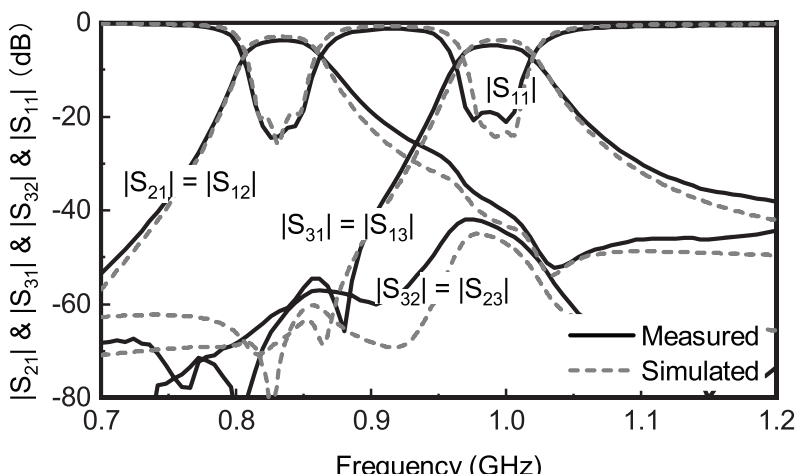

(a)

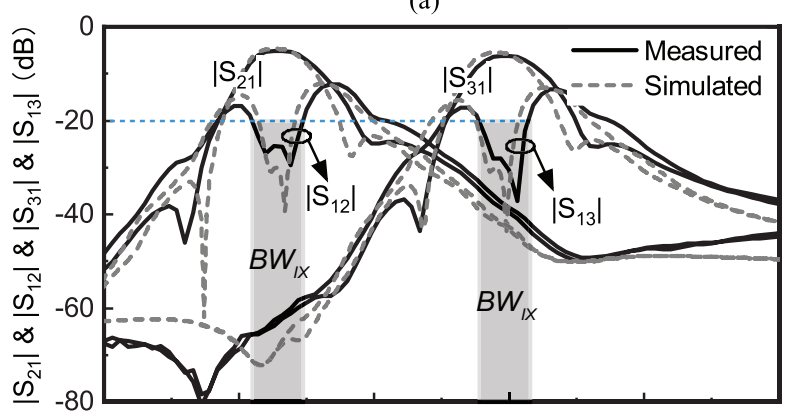

(b)

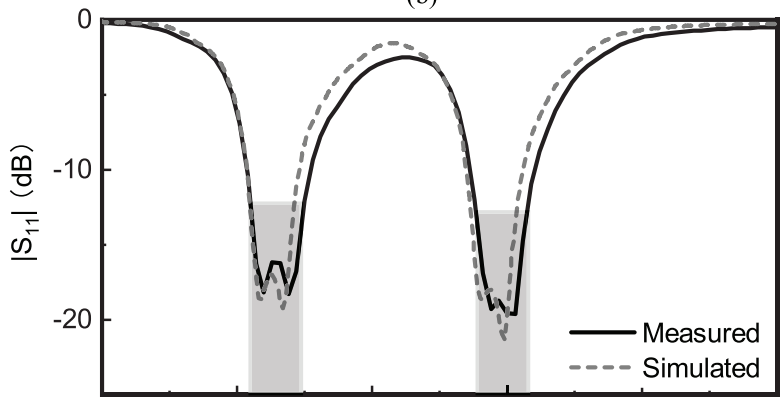

(c)

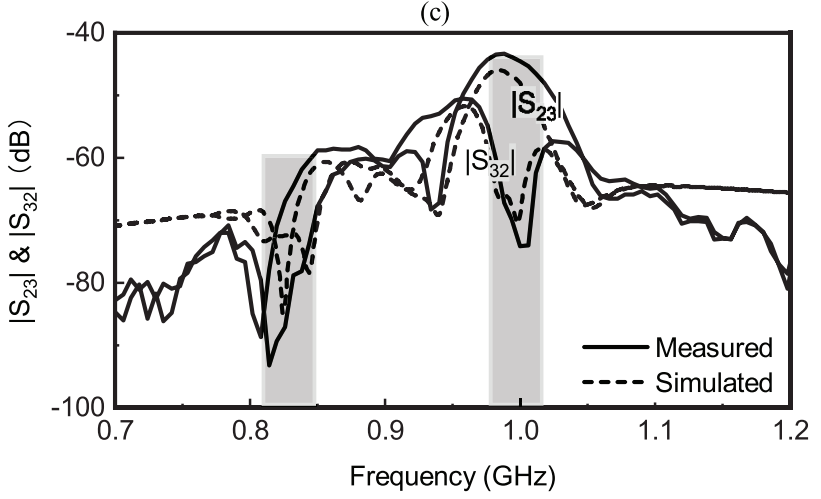

(d)

Fig. 10. Results of the 3-pole non-reciprocal microstrip diplexer. (a) Static responses. (b) $\left|S_{21}\right|,\left|S_{12}\right|,\left|S_{31}\right|$, and $\left|S_{13}\right|$ of the non-reciprocal diplexer. (c) $\left|S_{11}\right|$ of the non-reciprocal diplexer. (d) $\left|S_{23}\right|$ and $\left|S_{32}\right|$ of the non-reciprocal diplexer.

each channel can be observed from Fig.10(b). As seen in Fig.10(b), channel 1 has a measured transmission insertion loss of $4.9 \mathrm{~dB}$. The backward isolation at the center frequency $829 \mathrm{MHz}$ is $25 \mathrm{~dB}$ and it is better than $20 \mathrm{~dB}$ in the range of $810-848 \mathrm{MHz}$, i.e., $B W_{I X}=38 \mathrm{MHz}$ for channel 1 . Channel 2 has a measured transmission insertion loss of $6.1 \mathrm{~dB}$ [Fig.10(b)]. The backward isolation at the center frequency
$997 \mathrm{MHz}$ is $27.7 \mathrm{~dB}$ and it is better than $20 \mathrm{~dB}$ in the range of $978-1015 \mathrm{MHz}$, i.e., $B W_{I X}=37 \mathrm{MHz}$ for channel 2. The measured return loss over the $20-\mathrm{dB}$ isolation band is shown to be better than $12.1 \mathrm{~dB}$ for channel 1 and better than $13.2 \mathrm{~dB}$ for channel 2 [Fig.10(c)]. Higher than 43.5-dB output port isolation is maintained in Fig. 10(d).

Compared to the static results of Fig. 10(a), we can observe an additional insertion loss of $1.1 \mathrm{~dB}$ and $1.6 \mathrm{~dB}$ for channel 1 and channel 2, respectively. This is due to the signal conversion loss [35]. Future works can be done to lower the insertion loss by using either high- $Q$ resonators [42] or differential circuit configurations [29].

\section{Frequency Tunability}

Frequency tunability is of great interest to future wireless communications and sensing systems for better utilization of the limited frequency spectrum resources. For the filters presented in [36], because the true incremental modulation phase $\Delta \varphi$ across the varactors depends not only on the incremental phase of modulation sources but also on the unequal modulation feed lines. As a result, it is hard to reconfigure the non-reciprocity frequency. In contrast, $\Delta \varphi$ in the proposed work is independent of the modulation feed lines which are the same for all the modulation sources. Hence, the optimum value of $\Delta \varphi=45^{\circ}$ can always be satisfied for different $f_{m}$ by simply setting the incremental phase of external modulation sources to $45^{\circ}$. Accordingly, by changing the bias $d c$ voltage $\left(V_{d c}\right)$ and modulation parameters $\left(f_{m}\right.$ and $\left.V_{m}\right)$, the proposed non-reciprocal filter and diplexer can be tuned over a wide range in terms of the non-reciprocity frequency. Fig.111(a)-(c) in the next page show the frequency tuning performance of the 3-pole non-reciprocal filter. Although only a few discrete states are shown, the non-reciprocal filter can be continuously tuned. Specifically, the 3-pole non-reciprocal filter can be tuned from $885 \mathrm{MHz}$ to $1031 \mathrm{MHz}$, while maintaining an isolation larger than $20 \mathrm{~dB}$, a passband return loss better than $10.9 \mathrm{~dB}$, and a close-to-constant 20-dB isolation bandwidth of $44 \mathrm{MHz}$. Two distinct isolation poles in Fig.11(b) and two reflection poles in Fig.11(c) are observed in all the reconfigured states.

The tuning parameters $\left(V_{d c}, V_{m}, \Delta \varphi\right.$, and $\left.f_{m}\right)$ and critical performances of the tunable non-reciprocal filter are listed in Table $\mathbb{I}$. The insertion loss is lower at higher frequencies because the varactor quality factor is higher at smaller capacitance. This is generally observed in other solid-state varactor based tunable circuits [43], [44].

In a similar manner, the two channels of the proposed non-reciprocal diplexer can also be tuned. Take channel 1 for example, a tuning range of $815-851 \mathrm{MHz}$ is achieved by varying the $d c$ voltage and modulation parameters of channel 1 [Fig.11(d) and (e)]. There is only a slight loading effect on the matching of channel 2 when channel 1 is tuned [Fig.11](d)(f)]. This shows that the two channels of the proposed diplexer can be independently optimized and modulated, i.e., channel 2 can also be tuned without affecting channel 1. Several state parameters of the tunable non-reciprocal diplexer are also summarized in Table. I] Only channel 1 is characterized because channel 2 under the three tuning states has essentially the same characteristics as described in Section 


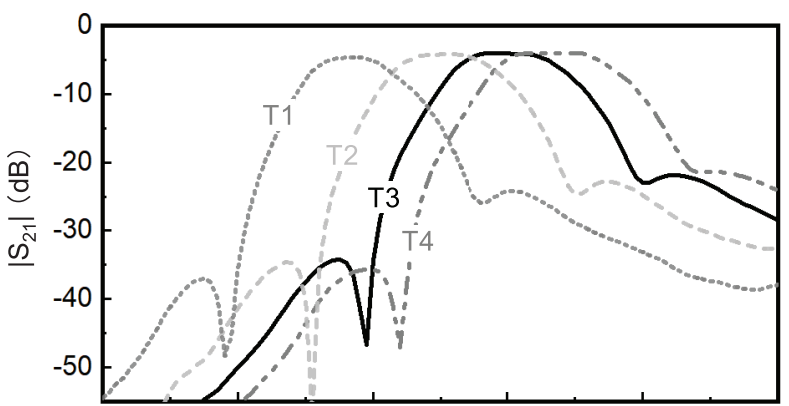

(a)

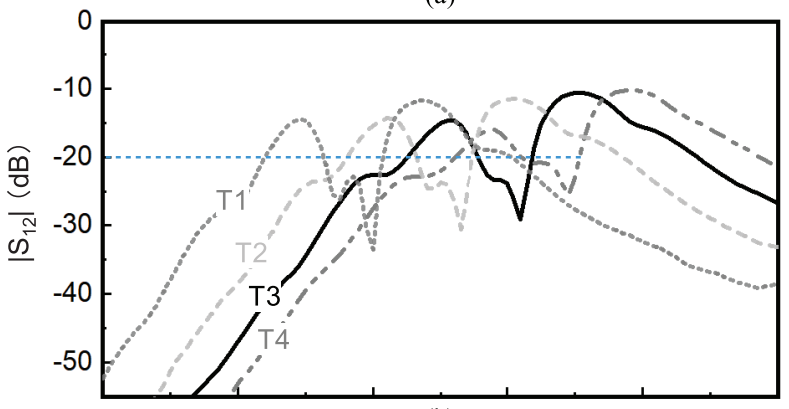

(b)

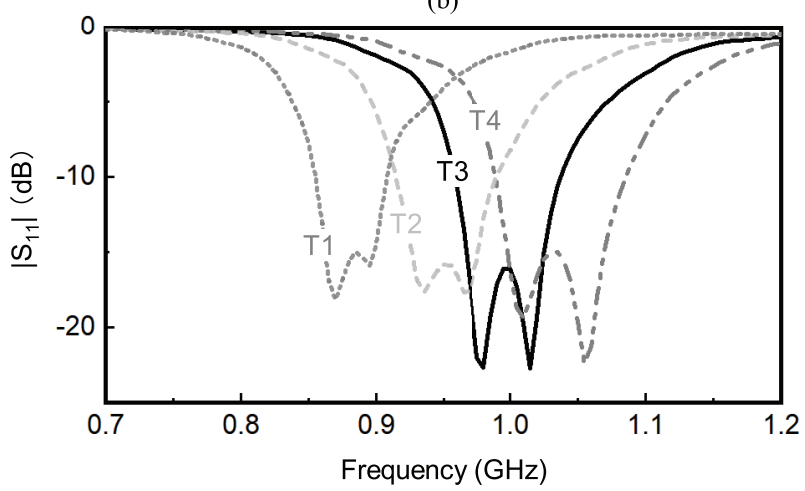

(c)

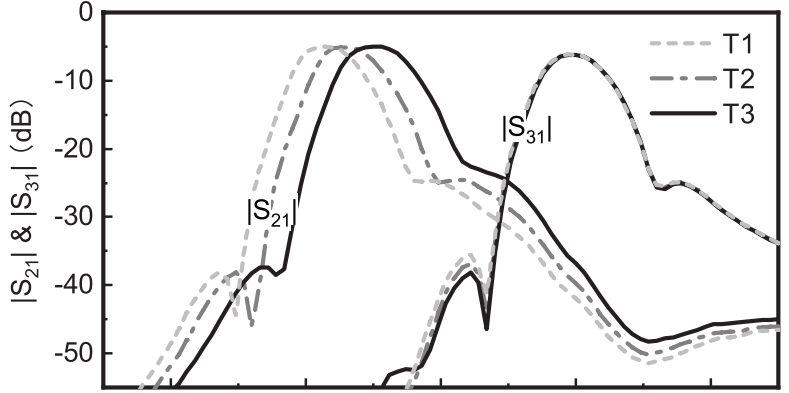

(d)

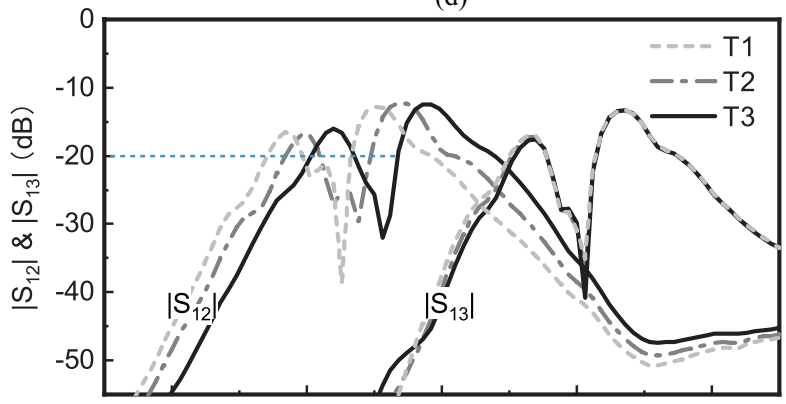

(e)

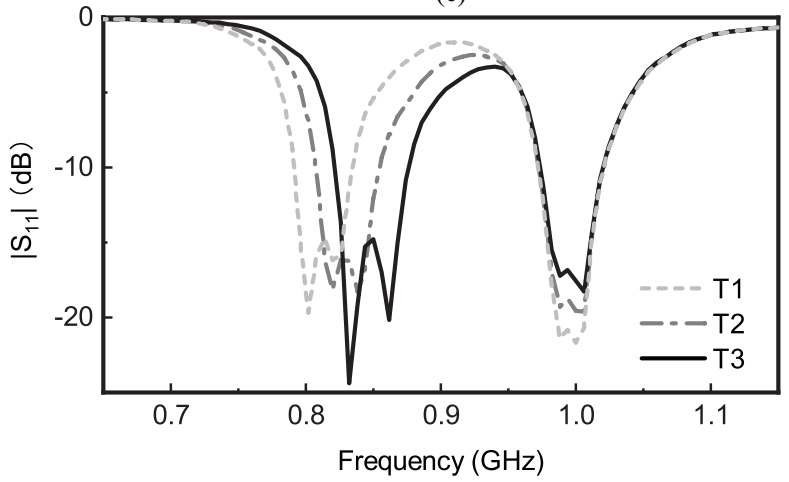

(f)

Fig. 11. Measured frequency tuning characteristics. (a), (b), and (c) are the reconfigured $\left|S_{21}\right|,\left|S_{12}\right|$, and $\left|S_{11}\right|$, respectively, of the 3-pole non-reciprocal filter, (d) is the reconfigured $\left|S_{21}\right|$ and $\left|S_{31}\right|$ of the non-reciprocal diplexer, (e) is the reconfigured $\left|S_{12}\right|$ and $\left|S_{13}\right|$ of the non-reciprocal diplexer, and (f) is the reconfigured $\left|S_{11}\right|$ of the non-reciprocal diplexer.

TABLE I

List of Measured 3-Pole Non-Reciprocal Microstrip Filter And Diplexer Parameters

\begin{tabular}{lllllllllll}
\hline Circuit & State & $V_{d c}$ & $V_{m}$ & $f_{m}$ & $\Delta \varphi$ & $f_{0}$ & IL & IX* & $B W_{I X}$ & $\mathrm{RL}$ \\
\hline \multirow{2}{*}{ Filter } & $\mathrm{T} 1$ & $3.8 \mathrm{~V}$ & $0.5 \mathrm{~V}$ & $60 \mathrm{MHz}$ & $45^{\circ}$ & $885 \mathrm{MHz}$ & $4.6 \mathrm{~dB}$ & $22.8 \mathrm{~dB}$ & $44 \mathrm{MHz}$ & $>10.9 \mathrm{~dB}$ \\
& $\mathrm{~T} 2$ & $4.5 \mathrm{~V}$ & $0.66 \mathrm{~V}$ & $62 \mathrm{MHz}$ & $45^{\circ}$ & $954 \mathrm{MHz}$ & $4.2 \mathrm{~dB}$ & $23.7 \mathrm{~dB}$ & $44 \mathrm{MHz}$ & $>15.9 \mathrm{~dB}$ \\
& $\mathrm{~T} 3$ & $6.5 \mathrm{~V}$ & $1.5 \mathrm{~V}$ & $65 \mathrm{MHz}$ & $45^{\circ}$ & $1000 \mathrm{MHz}$ & $3.9 \mathrm{~dB}$ & $23.2 \mathrm{~dB}$ & $42 \mathrm{MHz}$ & $>16.1 \mathrm{~dB}$ \\
& $\mathrm{~T} 4$ & $7.5 \mathrm{~V}$ & $2.1 \mathrm{~V}$ & $68 \mathrm{MHz}$ & $45^{\circ}$ & $1031 \mathrm{MHz}$ & $3.9 \mathrm{~dB}$ & $20.9 \mathrm{~dB}$ & $43 \mathrm{MHz}$ & $>15.1 \mathrm{~dB}$ \\
\hline \multirow{2}{*}{ Diplexer\# } & $\mathrm{T} 1$ & $6.5 \mathrm{~V}$ & $0.75 \mathrm{~V}$ & $42 \mathrm{MHz}$ & $45^{\circ}$ & $815 \mathrm{MHz}$ & $5.0 \mathrm{~dB}$ & $21.4 \mathrm{~dB}$ & $37 \mathrm{MHz}$ & $>11.4 \mathrm{~dB}$ \\
& $\mathrm{~T} 2$ & $5.9 \mathrm{~V}$ & $0.85 \mathrm{~V}$ & $42.5 \mathrm{MHz}$ & $45^{\circ}$ & $829 \mathrm{MHz}$ & $4.9 \mathrm{~dB}$ & $25.6 \mathrm{~dB}$ & $38 \mathrm{MHz}$ & $>12.1 \mathrm{~dB}$ \\
& $\mathrm{~T} 3$ & $5.6 \mathrm{~V}$ & $0.93 \mathrm{~V}$ & $43 \mathrm{MHz}$ & $45^{\circ}$ & $851 \mathrm{MHz}$ & $4.8 \mathrm{~dB}$ & $26.6 \mathrm{~dB}$ & $34 \mathrm{MHz}$ & $>15.1 \mathrm{~dB}$ \\
\hline
\end{tabular}

IL: minimum insertion loss of the forward transmission $\left|S_{21}\right|$.

IX*: backward isolation at $f_{0}$.

$B W_{I X}: 20-\mathrm{dB}$ isolation bandwidth.

RL: return loss over the $20-\mathrm{dB}$ isolation band;

\#: only channel 1 is characterized while channel 2 is unchanged.

Note that frequency tunability has been reported in STMbased circulators in [21] and [29]. However, only single- resonator response of one isolation pole is reported in these works. Due to the large group delay variations around the 
center frequency, single-resonator circuits generally present challenges to the system design. Although a circulator of two isolation poles is reported in [28], frequency tunability is not feasible due to the complicated biasing network and the fixedfrequency static resonators used in its design.

We would like to remark that larger $f_{m}$ as well as higher $V_{m}$ in Table.I are used for achieving non-reciprocity at higher frequency. This is because the corresponding filter has wider static transmission bandwidth $B W$ when the resonator frequency $f_{0}$ is increased. This is due to the frequency dependent nature of the coupling structures [45]. To have wider tunable range of constant passband bandwidth $B W$, frequency-curve shape design method in [46] and coupling matrix based extraction technique in [47] have been newly developed. Such ideas may be used in the future to improve the tuning performance of the proposed non-reciprocal circuits.

\section{Comparison With Previous Works}

At the time of this writing, only a few works on STMbased non-reciprocal filters are reported in the literature [35], [36], [48], [49]. In [35], we first presented the concept of using STM to create strong in-band non-reciprocity in an inline coupled-resonator filter. A lumped-element non-reciprocal filter was demonstrated at a center frequency of $200 \mathrm{MHz}$ with a minimum forward insertion loss of $1.5 \mathrm{~dB}$, an in-band reverse isolation better than $20 \mathrm{~dB}$, and an in-band return loss better than $15 \mathrm{~dB}$. Subsequently, a time-varying coupling matrix formulation was developed to analyze such filters [36]. A demonstration was provided with distributed non-reciprocal filters using microstrip time-modulated resonators. The $f_{m}$ $f_{R F}$ relied solely on large inductors. In addition, the true incremental modulation phase over the varactors depended not only on the incremental phase between modulation sources but also on the unequal modulation feed lines at each modulation port. This resulted in distorted modulation phases and nonoptimal frequency responses. Only one isolation pole was generated in the 3-pole example, limiting the isolation bandwidth. Furthermore, the closely spaced resonators brought undesired cross-coupling effect which caused significant deterioration on the upper band-edge transmission.

In this work, based on the proposed time-modulated $\lambda_{g} / 2$ resonator, a 3-pole non-reciprocal filter of simplified biasing strategy and improved performances is presented. Wideband $30-\mathrm{dB} f_{m}-f_{R F}$ isolation is achieved by loading the modulation sources to the center of $\lambda_{g} / 2$ resonators where an inherent voltage null exists for the fundamental resonance. Both the $\mathrm{RF}$ resonators and modulation circuits are integrated on top of the substrate and an intact ground is maintained to avoid interference especially when multiple RF circuits or elements are stacked or integrated together. Owing to this superior biasing design, improved non-reciprocal filtering responses with two isolation poles and two reflection poles are achieved. Lastly, the ring-type resonators used in this work implements a true inline-coupled topology [Fig.6(b)], which in return realizes a passband transmission with small insertion loss variation and no band-edge deterioration.

We also note that non-reciprocity in time-modulated coupled-resonator networks has been reported in [48] and
[49]. Similar to our findings in [35], Shen et al. showed that STM modulation of two coupled resonators can generate nonreciprocity but with deteriorated forward transmission [48]. In comparison, we showed that at least a 3-pole network is needed to create strong non-reciprocity while maintaining good transmission and reflection characteristics [35]. Indeed, stronger non-reciprocity was achieved in a high-order network. However, only one isolation pole is observed even for a 13 order STM network [49]. The main reason is that a suboptimal modulation phase of $\Delta \varphi=90^{\circ}$ was used. In contrast, the non-reciprocal filter in this work verifies that by applying proper modulation of $\Delta \varphi=45^{\circ}$ and $f_{m} \simeq B W$ [35], highorder non-reciprocity of multiple isolation poles (two isolation pole in this paper) can be obtained.

\section{CONCLUSIONS}

This paper introduces novel designs of distributed nonreciprocal filter and diplexer using time-modulated microstrip $\lambda_{g} / 2$ resonators. Wideband high $f_{m}-f_{R F}$ isolation is obtained by loading modulation source to the RF resonator at the central voltage null point through a single biasing inductor. The demonstrated filter and diplexer examples are of simple circuit configurations and achieve excellent non-reciprocal filtering performances. The effectiveness of the proposed design ideas is well validated by the close agreement between measurement and simulation results. In addition, reconfigured non-reciprocal characteristics of multiple isolation poles are readily illustrated by varying the reversely biased $d c$ voltage and modulation waves. The work presented in this paper advances the state-ofthe-art non-magnetic non-reciprocal devices at RF frequencies and paves a way to tunable function integrated microwave devices.

\section{REFERENCES}

[1] D. Bharadia, E. McMilin, and S. Katti, "Full duplex radios," in Proceedings of the ACM SIGCOMM 2013 Conference on SIGCOMM, ser. SIGCOMM '13. New York, NY, USA: ACM, 2013, pp. 375-386.

[2] A. Sabharwal, P. Schniter, D. Guo, D. W. Bliss, S. Rangarajan, and R. Wichman, "In-band full-duplex wireless: Challenges and opportunities," IEEE Journal on Selected Areas in Communications, vol. 32, no. 9, pp. 1637-1652, Sep. 2014.

[3] K. E. Kolodziej, B. T. Perry, and J. S. Herd, "In-band full-duplex technology: Techniques and systems survey," IEEE Trans. Microw. Theory Techn., vol. 67, no. 7, pp. 3025-3041, Jul. 2019.

[4] J. Zhou, N. Reiskarimian, J. Diakonikolas, T. Dinc, T. Chen, G. Zussman, and H. Krishnaswamy, "Integrated full duplex radios," IEEE Commun. Mag., vol. 55, no. 4, pp. 142-151, Apr. 2017.

[5] N. Reiskarimian, M. B. Dastjerdi, J. Zhou, and H. Krishnaswamy, "Analysis and design of commutation-based circulator-receivers for integrated full-duplex wireless," IEEE J. Solid-State Circuits, vol. 53, no. 8, pp. 2190-2201, Aug. 2018.

[6] A. Kord, D. L. Sounas, and A. Alú, "Achieving full-duplex communication: Magnetless parametric circulators for full-duplex communication systems," IEEE Microw. Mag., vol. 19, no. 1, pp. 84-90, Jan. 2018.

[7] C. E. Fay and R. L. Comstock, "Operation of the ferrite junction circulator," IEEE Trans. Microw. Theory Techn., vol. 13, no. 1, pp. 1527, Jan. 1965.

[8] S. A. Oliver, P. Shi, N. E. McGruer, C. Vittoria, W. Hu, H. How, S. W. McKnight, and P. M. Zavracky, "Integrated self-biased hexaferrite microstrip circulators for millimeter-wavelength applications," IEEE Trans. Microw. Theory Techn., vol. 49, no. 2, pp. 385-387, Feb. 2001.

[9] S. Tanaka, N. Shimomura, and K. Ohtake, "Active circulators: The realization of circulators using transistors," Proc. IEEE, vol. 53, no. 3 , pp. 260-267, Mar. 1965. 
[10] T. Kodera, D. L. Sounas, and C. Caloz, "Magnetless nonreciprocal metamaterial (MNM) technology: Application to microwave components," IEEE Trans. Microw. Theory Techn., vol. 61, no. 3, pp. 1030-1042, Mar. 2013.

[11] J. Chang, J. Kao, Y. Lin, and H. Wang, "Design and analysis of 24$\mathrm{GHz}$ active isolator and quasi-circulator," IEEE Trans. Microw. Theory Techn., vol. 63, no. 8, pp. 2638-2649, Aug. 2015.

[12] G. Carchon and B. Nanwelaers, "Power and noise limitations of active circulators," IEEE Trans. Microw. Theory Techn., vol. 48, no. 2, pp. 316-319, Feb. 2000.

[13] L. Fan, J. Wang, L. T. Varghese, H. Shen, B. Niu, Y. Xuan, A. M. Weiner, and M. Qi, "An all-silicon passive optical diode," Science, vol. 335, no. 6067, pp. 447-450, 2012.

[14] Y. Shi, Z. Yu, and S. Fan, "Limitations of nonlinear optical isolators due to dynamic reciprocity," Nature Photon., vol. 9, p. 388392, May 2015.

[15] D. L. Sounas, J. Soric, and A. Alú, "Broadband passive isolators based on coupled nonlinear resonances," Nature Electron., vol. 1, pp. 113-119, Feb. 2018.

[16] N. Reiskarimian, A. Nagulu, T. Dinc, and H. Krishnaswamy, "Nonreciprocal electronic devices: A hypothesis turned into reality," IEEE Microw. Mag., vol. 20, no. 4, pp. 94-111, Apr. 2019.

[17] S. Qin, Q. Xu, and Y. E. Wang, "Nonreciprocal components with distributedly modulated capacitors," IEEE Trans. Microw. Theory Techn., vol. 62 , no. 10 , pp. $2260-2272$, Oct. 2014.

[18] S. Taravati, "Self-biased broadband magnet-free linear isolator based on one-way space-time coherency," Phys. Rev. B, vol. 96, p. 235150, Dec. 2017.

[19] D. L. Sounas, C. Caloz, and A. Alú, "Giant non-reciprocity at the subwavelength scale using angular momentum-biased metamaterials," Nature Commun., vol. 4, p. 2407, Sep. 2013.

[20] A. Kamal, J. Clarke, and M. H. Devoret, "Noiseless non-reciprocity in a parametric active device," Nature Phys., vol. 7, p. 311315, Jan. 2011.

[21] N. A. Estep, D. L. Sounas, J. Soric, and A. Alú, "Magnetic-free nonreciprocity and isolation based on parametrically modulated coupledresonator loops," Nature Phys., vol. 10, pp. 923-927, Dec. 2014.

[22] N. A. Estep, D. L. Sounas, and A. Alú, "Magnetless microwave circulators based on spatiotemporally modulated rings of coupled resonators," IEEE Trans. Microw. Theory Techn., vol. 64, no. 2, pp. 502-518, Feb. 2016.

[23] A. Kord, D. L. Sounas, and A. Alú, "Magnet-less circulators based on spatiotemporal modulation of bandstop filters in a delta topology," IEEE Trans. Microw. Theory Techn., vol. 66, no. 2, pp. 911-926, Feb. 2018.

[24] M. M. Torunbalci, T. J. Odelberg, S. Sridaran, R. C. Ruby, and S. A Bhave, "An FBAR circulator," IEEE Microw. Wireless Compon. Lett., vol. 28, no. 5, pp. 395-397, May 2018.

[25] G. Michetti, C. Cassella, F. Pop, M. Pirro, A. Kord, D. Sounas, A. Alú, and M. Rinaldi, "A quasi-LTI frequency-selective SAW circulator," in 2018 IEEE International Ultrasonics Symposium (IUS), Oct. 2018, pp. 206-212.

[26] Y. Yu, G. Michetti, A. Kord, D. Sounas, F. V. Pop, P. Kulik, M. Pirro, Z. Qian, A. Alú, and M. Rinaldi, "Magnetic-free radio frequency circulator based on spatiotemporal commutation of MEMS resonators," in 2018 IEEE Micro Electro Mechanical Systems (MEMS), Jan. 2018 pp. $154-157$.

[27] M. Nafe, M. N. Hasan, H. Reggad, D. Kuzmenko, J. Chen, and X. Liu, "Magnetic-free circulator based on spatio-temporal modulation implemented via switched capacitors for full duplex communication," in 2018 USNC-URSI Radio Science Meeting (Joint with AP-S Symposium), Jul. 2018, pp. 119-120.

[28] A. Kord, D. L. Sounas, Z. Xiao, and A. Alú, "Broadband cyclicsymmetric magnetless circulators and theoretical bounds on their bandwidth," IEEE Trans. Microw. Theory Techn., vol. 66, no. 12, pp. 54725481, Dec. 2018.

[29] A. Kord, D. L. Sounas, and A. Alú, "Pseudo-linear time-invariant magnetless circulators based on differential spatiotemporal modulation of resonant junctions," IEEE Trans. Microw. Theory Techn., vol. 66, no. 6, pp. 2731-2745, Jun. 2018.

[30] N. Reiskarimian and H. Krishnaswamy, "Magnetic-free non-reciprocity based on staggered commutation," Nature Commun., vol. 7, p. 11217, Apr. 2016.

[31] T. Dinc, M. Tymchenko, A. Nagulu, D. Sounas, A. Alú, and H. Krishnaswamy, "Synchronized conductivity modulation to realize broadband lossless magnetic-free non-reciprocity," Nature Commun., vol. 8, p. 795, Oct. 2017.

[32] T. Dinc, A. Nagulu, and H. Krishnaswamy, "A millimeter-wave nonmagnetic passive SOI CMOS circulator based on spatio-temporal con- ductivity modulation," IEEE J. Solid-State Circuits, vol. 52, no. 12, pp. 3276-3292, Dec. 2017

[33] A. Nagulu, T. Dinc, Z. Xiao, M. Tymchenko, D. L. Sounas, A. Alú, and H. Krishnaswamy, "Nonreciprocal components based on switched transmission lines," IEEE Trans. Microw. Theory Techn., vol. 66, no. 11, pp. 4706-4725, Nov. 2018.

[34] R. Lu, T. Manzaneque, Y. Yang, L. Gao, A. Gao, and S. Gong, "A radio frequency nonreciprocal network based on switched acoustic delay lines," IEEE Trans. Microw. Theory Techn., vol. 67, no. 4, pp. 15161530, Apr. 2019.

[35] X. Wu, X. Liu, M. D. Hickle, D. Peroulis, J. S. Gómez-Díaz, and A. Álvarez Melcón, "Isolating bandpass filters using time-modulated resonators," IEEE Trans. Microw. Theory Techn., vol. 67, no. 6, pp. 2331-2345, Jun. 2019.

[36] A. Álvarez Melcón, X. Wu, J. Zang, X. Liu, and J. S. Gómez-Díaz, "Coupling matrix representation of nonreciprocal filters based on time modulated resonators," IEEE Trans. Microw. Theory Techn., vol. 67, no. 12, pp. 4751-4763, Dec. 2019.

[37] X. Wu, M. Nafe, A. Álvarez Melcón, J. S. Gómez-Díaz, and X. Liu, "A non-reciprocal microstrip bandpass filter based on spatio-temporal modulation," in 2019 IEEE MTT-S International Microwave Symposium (IMS), Jun. 2019, pp. 9-12.

[38] SMV123x series: Hyperabrupt junction tuning varactors, Skyworks Solutions, Inc., Nov. 2018.

[39] J.-S. Hong and M. J. Lancaster, Microstrip Filters for RF / Microwave Applications. Wiley, 2001.

[40] K. Buisman, C. Huang, A. Akhnoukh, M. Marchetti, L. C. N. de Vreede, L. E. Larson, and L. K. Nanver, "Varactor topologies for RF adaptivity with improved power handling and linearity," in 2007 IEEE/MTT-S International Microwave Symposium, June 2007, pp. 319-322.

[41] M. A. El-Tanani and G. M. Rebeiz, "A two-pole two-zero tunable filter with improved linearity," IEEE Trans. Microw. Theory Techn., vol. 57, no. 4, pp. 830-839, Apr. 2009.

[42] R. R. Mansour, F. Huang, S. Fouladi, W. D. Yan, and M. Nasr, "HighQ tunable filters: challenges and potential," IEEE Microwave Mag., vol. 15 , no. 5 , pp. $70-82$, Jul. 2014.

[43] A. Anand, J. Small, D. Peroulis, and X. Liu, "Theory and design of octave tunable filters with lumped tuning elements," IEEE Trans. Microw. Theory Techn., vol. 61, no. 12, pp. 4353-4364, Dec. 2013.

[44] X. Liu, L. P. Katehi, W. J. Chappell, and D. Peroulis, "High-Q tunable microwave cavity resonators and filters using SOI-based RF MEMS tuners," J. Microelectromech. Syst., vol. 19, no. 4, pp. 774-784, 2010.

[45] Y. Cho and G. M. Rebeiz, "Two- and four-pole tunable 0.7-1.1GHz bandpass-to-bandstop filters with bandwidth control," IEEE Trans. Microw. Theory Techn., vol. 62, no. 3, pp. 457-463, Mar. 2014.

[46] M. Ohira, S. Hashimoto, Z. Ma, and X. Wang, "Coupling-matrixbased systematic design of single-DC-bias-controlled microstrip higher order tunable bandpass filters with constant absolute bandwidth and transmission zeros," IEEE Trans. Microw. Theory Techn., vol. 67, no. 1 , pp. 118-128, Jan. 2019.

[47] D. Lu, M. Yu, N. S. Barker, Z. Li, W. Li, and X. Tang, "Advanced synthesis of wide-tuning-range frequency-adaptive bandpass filter with constant absolute bandwidth," IEEE Trans. Microw. Theory Techn., vol. 67, no. 11, pp. 4362-4375, Nov. 2019.

[48] C. Shen, J. Li, Z. Jia, Y. Xie, and S. A. Cummer, "Nonreciprocal acoustic transmission in cascaded resonators via spatiotemporal modulation," Phys. Rev. B, vol. 99, p. 134306, Apr. 2019.

[49] C. W. Peterson, W. A. Benalcazar, M. Lin, T. L. Hughes, and G. Bahl, "Strong nonreciprocity in modulated resonator chains through synthetic electric and magnetic fields," Phys. Rev. Lett., vol. 123, p. 063901, Aug. 2019. 